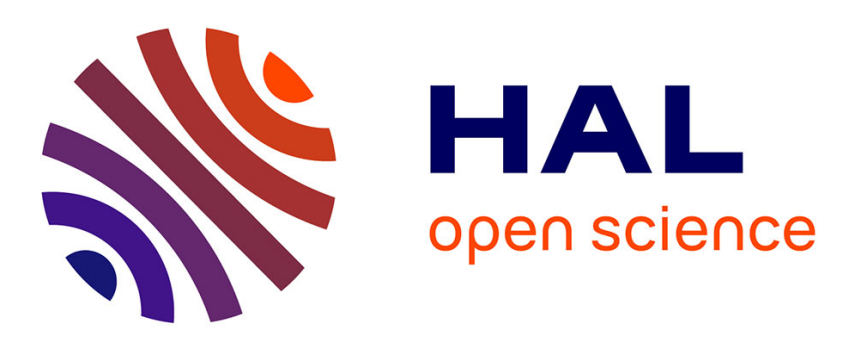

\title{
Modelling Mobile Object Activities Based on Trajectory Ontology Rules Considering Spatial Relationship Rules
}

Rouaa Wannous, Jamal Malki, Alain Bouju, Cécile Vincent

\section{To cite this version:}

Rouaa Wannous, Jamal Malki, Alain Bouju, Cécile Vincent. Modelling Mobile Object Activities Based on Trajectory Ontology Rules Considering Spatial Relationship Rules. 2013. hal-00917288

\author{
HAL Id: hal-00917288 \\ https://hal.science/hal-00917288
}

Submitted on 11 Dec 2013

HAL is a multi-disciplinary open access archive for the deposit and dissemination of scientific research documents, whether they are published or not. The documents may come from teaching and research institutions in France or abroad, or from public or private research centers.
L'archive ouverte pluridisciplinaire $\mathbf{H A L}$, est destinée au dépôt et à la diffusion de documents scientifiques de niveau recherche, publiés ou non, émanant des établissements d'enseignement et de recherche français ou étrangers, des laboratoires publics ou privés. 


\title{
Modelling Mobile Object Activities Based on Trajectory Ontology Rules Considering Spatial Relationship Rules
}

\author{
Rouaa WANNOUS ${ }^{1}$, Jamal MALKI ${ }^{1}$, Alain BOUJU ${ }^{1}$ and Cécile VINCENT ${ }^{2}$ \\ 1 Univ of La Rochelle, L3i laboratory, France \\ 2 Univ of La Rochelle, LIENSs laboratory, France \\ \{rwannous, jmalki, abouju, Cvincent\}@univ-lr.fr
}

\begin{abstract}
Several applications use devices and capture systems to record trajectories of mobile objects. To exploit these raw trajectories, we need to enhance them with semantic information. Temporal, spatial and domain related information are fundamental sources used to upgrade trajectories. The objective of semantic trajectories is to help users validating and acquiring more knowledge about mobile objects. In particular, temporal and spatial analysis of semantic trajectories is very important to understand the mobile object behaviour. This article proposes an ontology based modelling approach for semantic trajectories. This approach considers different and independent sources of knowledge represented by domain and spatial ontologies. The domain ontology represents mobile object activities as a set of rules. The spatial ontology represents spatial relationships as a set of rules. To achieve this approach, we need an integration between trajectory and spatial ontologies.
\end{abstract}

Keywords: Trajectory data modelling, Modelling activities, Ontology rules, Spatial data modelling.

\section{Introduction}

Over the last few years, there has been a huge collection of real-time data on mobile objects. These data are obtained by many kind of systems like GNSS ${ }^{3}$ (GPS4 ${ }^{4}$ or ARGOS 5 , phone location or RFID ${ }^{6}$. This opens new perspectives for developing applications, such as bird migration monitoring [14, daily trips of employees [19], military application [13] and marine mammal tracking [8]. The raw data captured, commonly called trajectories, traces moving objects from a departure point to a destination point as sequences of pairs (sample points captured, time of the capture). In [14, the authors give a general definition of a trajectory: A trajectory is the user defined record of the evolution of the position

\footnotetext{
${ }^{3}$ GNSS : Global Navigation Satellite System

${ }^{4}$ GPS: Global Positioning System

${ }^{5}$ ARGOS: Advanced Research and Global Observation Satellite

${ }^{6}$ RFID: Radio Frequency IDentification
} 
(perceived as a point) of an object that is moving in space during a given time interval in order to achieve a given goal. Raw trajectories contain neither contextual information about the displacement of a moving object nor its accomplished activities [1]. Semantic trajectories can be seen as a high-level data layer on raw trajectories [19]. Furthermore, it becomes necessary to provide mechanisms for storage, modelling, efficient analysis and knowledge extraction from these data to enable interoperability between systems and services. Ontologies have been proposed as a solution for modelling data with their semantic information. To exploit raw trajectories, we need other data sources. Temporal, spatial and domain related information are fundamental sources. In the continuation of our previous work [8], a domain ontology was constructed to model semantic trajectory concepts and domain rules. We focused on semantic annotations for seal trajectories activities. We discussed the temporal data dimension of trajectories. This approach takes into account the temporal data features from low-level to high-level trajectory modelling. We give an evaluation of our approach on generated and real data. In this work, we are interested in modelling mobile object activities while considering the spatial relationships.

Raw trajectories can be captured as sample points given by their coordinates with the time of capture. So, a trajectory can be considered as spatio-temporal data. From this point of view, we can consider spatio-temporal data models to represent trajectory data. Nevertheless, these models do not propose specific support for a trajectory as a whole entity 512. Trajectory can also be considered from the point of view of the moving object. Moving object data models have been defined to represent trajectories [6]. Our approach models a trajectory by an RDF graph combining features from domain application and both spatio-temporal and moving objects data models. In this paper, we illustrate our proposal to integrate these three data models.

This paper is organized as follows. Section 2 summarizes some recent related work on semantic trajectories. Section 3 represents the domain application considered in this work. Section 4 details independently the ontological modelling approach: domain seal trajectory and spatial ontologies. Section 5 introduces the domain and spatial rules. Section 6 illustrates the integration between seal trajectory and spatial ontologies. Finally, Sect. 7 concludes this paper and presents some future prospects.

\section{Related work}

Data management techniques including modelling, indexing, inferencing and querying large spatio-temporal data have been actively investigated during the last decade [17977. Most of these techniques are only interested in raw trajectories [19 151]. The objective is to represent and query moving object trajectories. In [6], authors notice two data modelling points of view for trajectories: the conceptual modelling view and the moving objects view. Both need spatio-temporal data modelling and reasoning. 
Projects like GeoPKDD [4] and MODAP [10] emphasized the need to address and to use semantic data about moving objects for efficient trajectory analysis. For example, in [14, bird migration monitoring was analysed to get better understanding of bird behaviour. Scientists tried to answer queries such as: where, why and how long birds stop on their travels, the activities they engage during their stops, and which weather conditions the birds face during their flight. Considering these new requirements, new research has emerged offering data models that can be easily expanded taking into account semantic data. Thereby, a trajectory is seen as a user defined time-space function from a temporal interval to a space interval. To consider semantic trajectories, a conceptual view was defined by three main concepts: stops, moves, and begin-end of a trajectory [14. Each part contains a set of semantic data. Based on this conceptual model of trajectories, several studies have been proposed such as [12]. Moreover, in [19], authors designed a conceptual model of trajectories from low-level real-life GNSS data to different semantically abstracted levels. Their application concerned daily trips of employees from home to work and back.

Using ontologies as a model for semantic spatio-temporal data is a recent research field. In [15, authors worked on marine mammal tracking with the objective of understanding the behaviour of the animal by studying its activities. To model semantic trajectories, an ontological approach was defined to represent trajectory concepts. The ontologies constructed are formalised in RDF and OWL languages. This approach takes into account thematic and temporal rules 8 . In consequence, the inference mechanism was based on domain rules in addition to temporal and spatial rules defined as entailments. Moreover, in [9], authors worked on a military application domain with complex queries that require sophisticated inference methods. For this application, they presented an upper-level ontology defining a general hierarchy of thematic and spatial entity classes and associating relationships to connect these entity classes. They intended for application-specific domain ontologies in the thematic dimension to be integrated into the upper-level ontology through subclassing of appropriate classes and relationships. Consequently, the inference mechanism was based on several domain specific table functions and used only RDFS rules indexes.

Correspondingly, an integration between application domain ontology and spatial ontology led to the discovery of more semantics on trajectories. Furthermore, an ontological framework was produced in [18, composed of a modular ontology and its three component modules. The three following ontologies were integrated into a unique ontology by setting up rules between them to get more semantics:

1. Geometric trajectory ontology is a generic ontology that describes in particular the spatio-temporal features of a trajectory;

2. Geographic ontology describes the geographic objects;

3. The domain application ontology describes the thematic objects of the application. 


\section{Domain application}

Our modelling approach considers trajectories of seals. The data comes from the LIENS: $s^{7}$ (CNRS/University of La Rochelle) in collaboration with SMRU ${ }^{8}$. The captured spatio-temporal data of seal trajectories can be classified into three main states: haulout, cruise and dive [15]. In every state, there is a specific activity: resting, travelling and foraging, respectively. Based on these activities, we aim at answering queries, such as:

Example 1. seals foraging in a specific area

Analysing this query highlights the necessary of defining seal activities, such as foraging. Nevertheless, the spatial concepts representing area and the spatial relationship contains must be defined. Table 1 analyses the example query and illustrates the domain and spatial requirements.

Table 1. Domain, spatial concepts and rules needed for answering the example query

\begin{tabular}{llll}
\hline \multicolumn{2}{c}{ Concepts and rules } & Description \\
\hline \multirow{2}{*}{ Concepts } & Domain & Dive & specific part of the seal trajectory \\
\cline { 2 - 3 } Rules & Spatial & Polygon/Region & spatial concept for area \\
\cline { 2 - 3 } & Domain & Foraging & seal activity \\
\cline { 2 - 3 } & Spatial & Contains & $\begin{array}{l}\text { spatial relationship between the domain } \\
\text { and spatial concepts }\end{array}$ \\
\hline
\end{tabular}

\section{Ontology based modelling of trajectory}

The need of a spatial model and its relationships clearly appears from Table 1 . In this section, we consider independently trajectory and spatial data models.

\subsection{Trajectory ontology model}

The seal trajectory ontology, called owlsealtrajectory, is a result of a model transformation like in model-driven engineering approaches. The input of this transformation is the semantic seal trajectory model represented by a UML class diagram. Figure 1 presents an extract of this ontology, where:

- Seal is a mobile object. It represents the animal equipped with a tag;

- Sequence is captured in the form of temporal intervals.

- Trajectory is a logical form to represent a set of sequences;

- Activity is the semantic part representing seal activities for a sequence;

- Geosequence is the spatial part in seal trajectory ontology and can be Haulout, Cruise or Dive;

- Position is a point location of a geosequence. 


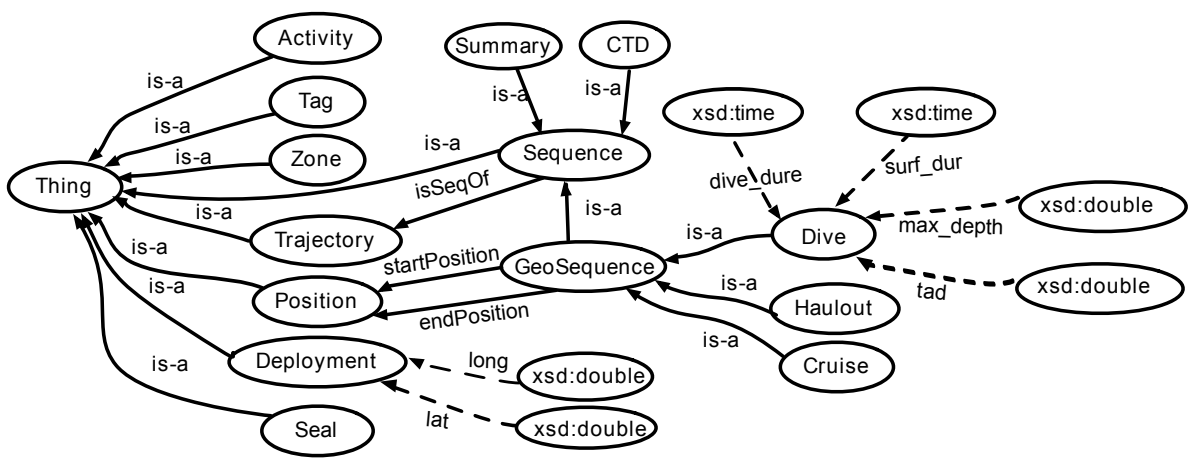

Fig. 1. An extract of owlsealtrajectory ontology

Besides these concepts, owlSealTrajectory defines relationships like:

- seqHasActivity is an object property between an activity and a sequence;

- startPosition, endPosition are object properties between a position and a geosequence. They represent start and end captured position of a geosequence;

- long, lat are data properties for the position of a captured point;

- dive_dur, sur_dur and max_depth are dive duration, surface duration and maximum depth of a dive, respectively;

- TAD is Time Allocation at Depth which defines the shape of a seal's dive 3.

\subsection{Spatial ontology model}

To model the spatial data dimension of a trajectory, we introduce a spatial ontology. In our approach, we choose owloGCSpatial ontology developed by Malki [7. This ontology is obtained by a model transformation. The input of this transformation is the spatial model represented by a UML class diagram proposed by Open Geospatial Consortium (OGC) [16]. An extract of the declarative part of this ontology is shown in Fig. 2 .

\section{$5 \quad$ Rule definition in the trajectory ontology}

Table 1 highlights the need of rules defined between ontology concepts: domain rules which are seal activities as well as spatial relationship rules.

\subsection{Domain seal trajectory rules}

Throughout the rules associated with the domain seal trajectory, we focus on seal activities. With our domain expert, we define four seal activities during their

\footnotetext{
${ }^{7}$ http://lienss.univ-larochelle.fr

${ }^{8}$ SMRU: Sea Mammal Research Unit- http://www.smru.st-and.ac.uk
} 


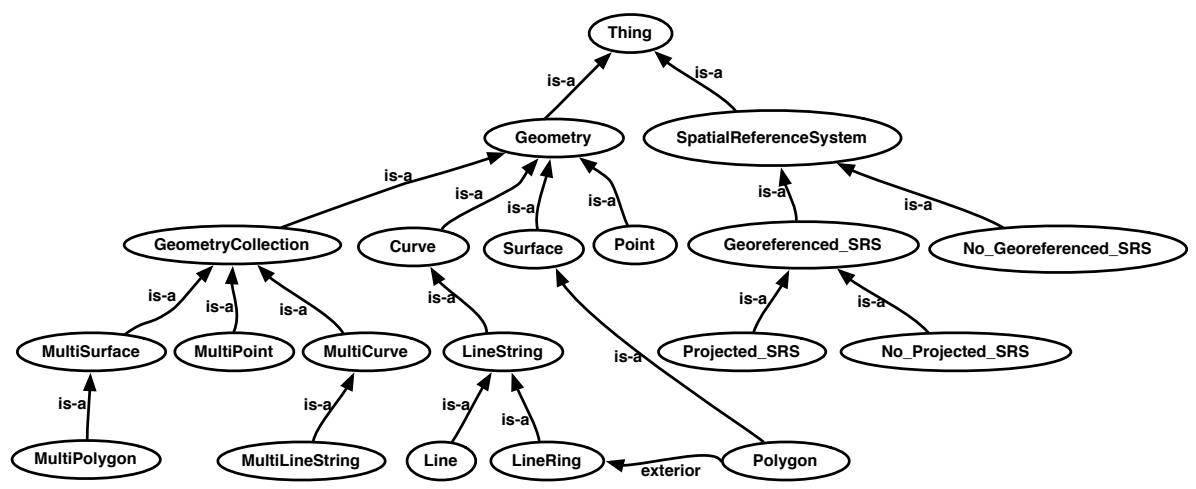

Fig. 2. A view of owlogCSpatial ontology

trajectory: travelling, resting, foraging and travelling-foraging. Figure 3 shows the declarative part of these activities. We implement them as an object relationship seqHasActivity between the sequence and activity concepts. The implementation of these rules is based on Table 2, This decision table shows the classification of seal activities based on parameters and considerations established by the domain expert. We use Oracle Semantic Data Store to implement these rules. We create the rule base sealActivities_rb to hold this implementation. Code 1.1 shows the implementation of foraging_rule: where maximum dive depth is more than 3 meters, TAD is bigger than 0.9 and less than 1 and finally, surface duration divided by dive duration is smaller than 0.5 .

Table 2. Decision table associated with seal activities

\begin{tabular}{llll}
\hline Rules & $\begin{array}{l}\text { Max dive } \\
\text { depth (meter) }\end{array}$ & $\begin{array}{l}\text { Dive shape } \\
\text { or TAD }\end{array}$ & $\begin{array}{l}\text { Surface ratio }= \\
\text { surface dur } / \text { dive dur }\end{array}$ \\
\hline Resting & $<10$ & all & $>0.5$ \\
\hline Travelling & $>3$ & $>0 \&<0.7$ & $<0.5$ \\
\hline Foraging & $>3$ & $>0.9 \&<1$ & $<0.5$ \\
\hline Travelling_Foraging & $>3$ & $>0.7 \&<0.9$ & $<0.5$ \\
\hline
\end{tabular}

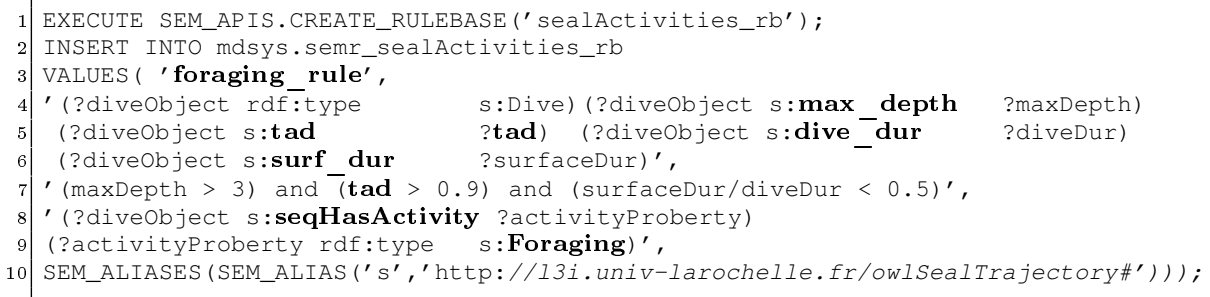

Code 1.1. Implementation of foraging rule 


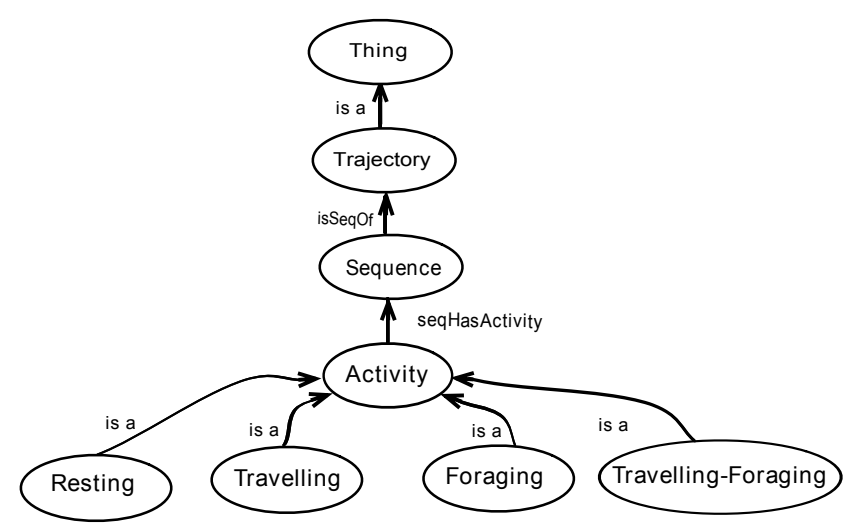

Fig. 3. Declarative part of seal activities

\subsection{Spatial relationship rules}

Spatial relationships are usually classified as topological, directional, and metric relationships. In this paper, we consider the topological relationships: Equals, Disjoint, Intersects, Touches, Crosses, Overlaps, AnyInteract, Within, and contains. We use Oracle Semantic Data Store to implement these relationships. For each spatial relationship, we associate an ontology rule in the rule base owlspatialonto_rb. For example, Code 1.2 presents the implementation of the imperative part of Contains_rule. In line 6, the property wkt represents the coordinate of the spatial objects. While we consider these ontology rules based on Oracle spatial layer, we implement a PL-SQL function called evalspatialRules. This function connects the spatial rules with the corresponding Oracle spatial operators, as shown in Fig. 4. Spatial Data Option SDO_ is the prefix for the implementations of Oracle spatial operators [11. Figure 6 illustrates the algorithm for calculating an inference for two spatial objects. For every two spatial objects, the inference procedure calls the spatial rules. The evalspatialRules function calls the corresponding Oracle spatial operator. The result is returned to the spatial rule to specify if there is a relationships between the two spatial objects. When calculating a new relationship, a new inference triple is generated and saved in an entailment.

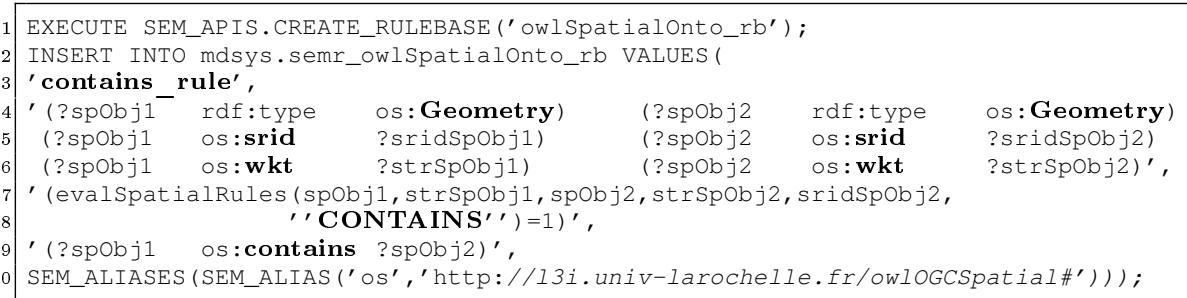

Code 1.2. Implementation of Contains spatial rule 


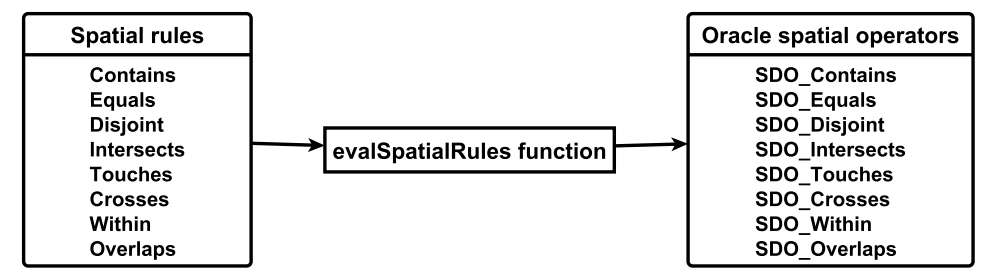

Fig. 4. Connect spatial rules with Oracle spatial operators

\section{Integrating trajectory and spatial ontologies}

The need of a semantic integration is fundamental while considering different and independent sources of information. For this integration, we are based on Position and GeoSequence concepts in seal trajectory ontology as mentioned in Sect. 4.1. The integration process of owlogCSpatial ontology with seal trajectory ontology follows these steps, as shown in Fig. 5

1. owlSealTrajectory:Position is mapped by the OWL statement owl: equivalentClass to owlogcspatial:Point.

2. owlSealTrajectory: Geosequence is mapped by OWL statement owl: equivalentClass to owlogCSpatial: Line.

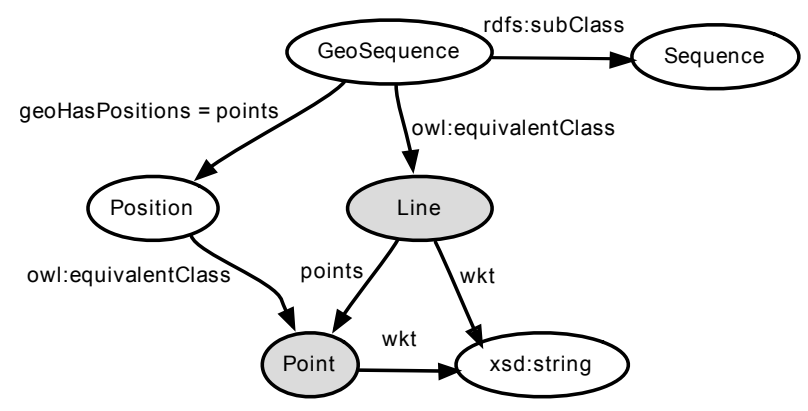

Fig. 5. Integrating owlSealTrajectory and owlogCSpatial ontologies

\section{Conclusion and future work}

Trajectories are usually available as raw data. Indeed, raw trajectories, collected by sensors, do not embed any kind of information about the travel of the moving object or about a possible interpretation of this travel. So, trajectory lacks semantics which is fundamental importance for its efficient use. In this work, 


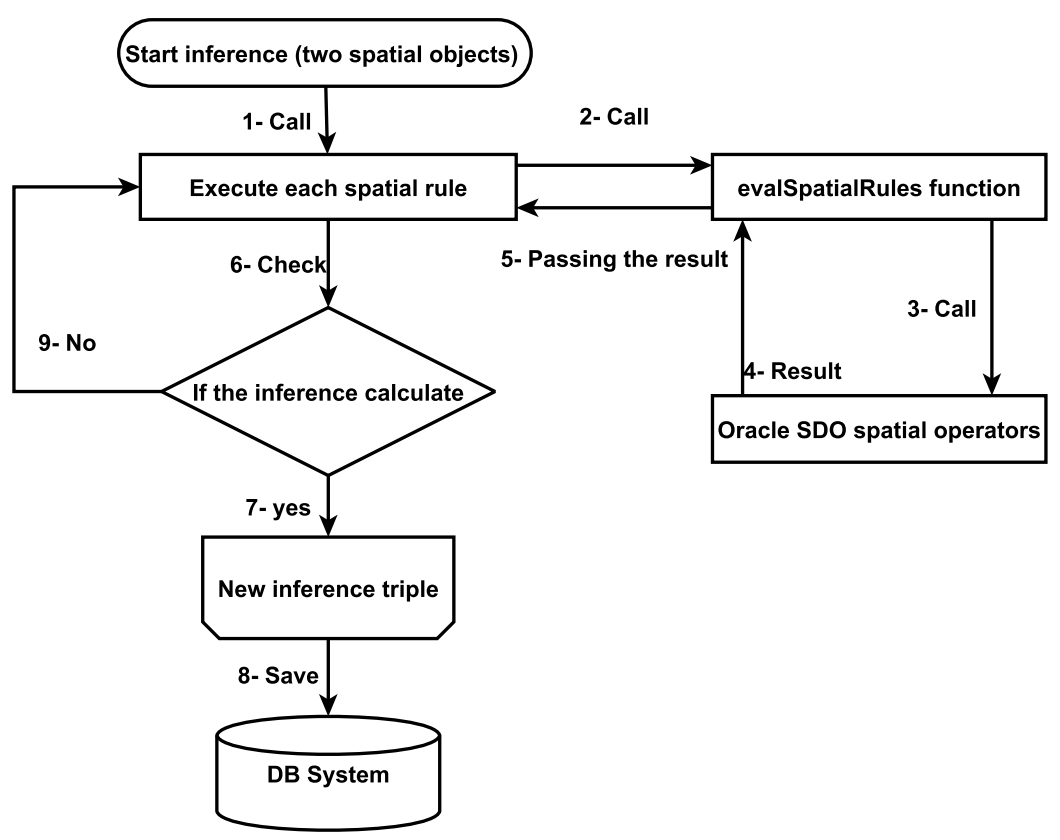

Fig. 6. Calculate the inference for two spatial objects

we present an ontological approach for modelling semantic trajectories. This approach considers the spatial characteristics of semantic trajectories. Based on the principle of reusing existing ontologies and considering different and independent sources of knowledge, we define an ontological integration approach to connect the domain and spatial ontologies. Throughout the defined ontologies, we implement the domain rules and spatial relationship rules.

In our future work, we will evaluate this approach on real data and we will compare results with other approaches. Moreover, we are highly interested in defining new notions of semantic trajectories and the integration of data mining algorithms with ontological rules.

\section{References}

1. M. Baglioni, J. Macedo, C. Renso, and M. Wachowicz. An ontology-based approach for the semantic modelling and reasoning on trajectories. In Advances in Conceptual Modeling - Challenges and Opportunities, volume 5232, pages 344-353. Springer Berlin/Heidelberg, 2008.

2. V. Bogorny, C. Heuser, and L. Alvares. A conceptual data model for trajectory data mining. In Geographic Information Sci., volume 6292, pages 1-15. Springer Berlin/Heidelberg, 2010. 
3. M. A. Fedak, P. Lovell, and S. M. Grant. Two approaches to compressing and interpreting time-depth information as collected by time-depth recorders and satellitelinked data recorders. Mar Mamm Sci, 17(1):94-110, 2001.

4. GeoPKDD. Geographic privacy-aware knowledge discovery and delivery. In Coordinator: KDDLAB, Knowledge Discovery nad Delivery Laboratory, ISTI-CNR and University of Pisa., 2005. http://www.geopkdd.eu/.

5. B. Huang and C. Claramunt. STOQL: An ODMG-based spatio-temporal object model and query language, 2002.

6. J. Macedo, C. Vangenot, W. Othman, N. Pelekis, E. Frentzos, B. Kuijpers, I. Ntoutsi, S. Spaccapietra, and Y. Theodoridis. Trajectory data models. In Mobility, Data Mining and Privacy, pages 123-150. Springer Berlin Heidelberg, 2008.

7. J. Malki, A. Bouju, and W. Mefteh. An ontological approach modeling and reasoning on trajectories. taking into account thematic, temporal and spatial rules. In TSI. Technique et Science Informatiques, volume 31/1-2012, pages 71-96, 2012.

8. J. Malki, R. Wannous, A. Bouju, and C. Vincent. Temporal reasoning in trajectories using an ontological modelling approach. Control and Cybernetics, 41:1-16, 2012.

9. P. Matthew. A framework to support spatial, temporal and thematic analytics over semantic web data. PhD thesis, Wright State Univ., 2008.

10. MODAP. Mobility, data mining and privacy, 2009. http://www.modap.org/.

11. Oracle. Oracle spatial developer's guide $11 \mathrm{~g}$ release 2 (11.2), 1996. http://docs.oracle.com/cd/E11882/.

12. C. Parent, S. Spaccapietra, and E. Zimanyi. Spatio-temporal conceptual models: Data structures + space + time. In Proceedings of the 7 th ACM international symposium on Advances in geographic information systems, pages 26-33. ACM, 1999.

13. M. Perry and W. S. University. A Framework to Support Spatial, Temporal and Thematic Analytics Over Semantic Web Data. Wright State University, 2008.

14. S. Spaccapietra, C. Parent, M. Damiani, J. Demacedo, F. Porto, and C. Vangenot. A conceptual view on trajectories. Data and Knowledge Engineering, 65(1):126146, 2008.

15. R. Wannous, J. Malki, A. Bouju, and C. Vincent. Time integration in semantic trajectories using an ontological modelling approach. In New Trends in Databases and Information Systems, volume 185 of Advances in Intelligent Systems and Computing, pages 187-198. Springer Berlin Heidelberg, 2013.

16. K. Werner and R. Martin. Open GIS consortium, inc, openGIS simple features specification for SQL, 1999.

17. Z. Yan, D. Chakraborty, C. Parent, S. Spaccapietra, and K. Aberer. SeMiTri: A framework for semantic annotation of heterogeneous trajectories. In Proceedings of the 14th International Conference on Extending Database Technology, pages 259270. ACM, 2011.

18. Z. Yan, J. Macedo, C. Parent, and S. Spaccapietra. Trajectory ontologies and queries. Transactions in GIS, 12(s1):75-91, 2008.

19. Z. Yan, C. Parent, S. Spaccapietra, and D. Chakraborty. A hybrid model and computing platform for spatio-semantic trajectories. In The Semantic Web: Research and Applications, pages 60-75. Springer Berlin/Heidelberg, 2010. 